\title{
An intrinsic behavioural approach to the double time axis paradox
}

\author{
Wenming Bian, Mark French and Harish Pillai
}

\begin{abstract}
Behavioural theory is typically developed on the double time axis. On the other hand it is known that there are intrinsic difficulties with double time axis theorems in the input output context. This paper illustrates how the behavioural approach avoids these intrinsic difficulties.
\end{abstract}

\section{INTRODUCTION}

In [3] it is shown that classical notions of stability and causality lead to problematic inconsistencies when inputoutput systems defined over a doubly infinite time axis are considered. On the other hand, behavioural theory [5], [8], [9], [10] has long been traditionally developed on the double time axis. The purpose of this paper is to revisit the GeorgiouSmith paradox from the vantage point of behavioural theory to see how a consistent 'double time axis' theory can be developed. In so doing, we will see that the behavioural approach avoids the paradox due to the inclusion of the 'free response' in the basic object of study (behaviours include autonomous sub-behaviours, contrast to the graph object in input-output theories which exclude 'free responses').

For concreteness, we include all the axiomatic definitions used in the behavioural development; note that this trajectory based treatment is deliberate and necessary: we want the results to be applied to systems which cannot be described by (finite dimensional) differential or difference behaviours as is common in most behavioural approaches. Indeed the basic example of study in this paper, namely the set of input/output pairs specified by the operator (2.1) does not form a differential behaviour. From a behavioural point of view ([5], [8], [9], [10]), the approach is especially fundamental. Much has been made of the intrinsic nature of behavioural definitions and the need for 'representation free' approaches.

\section{THE INTRINSIC DIFFICULTIES OF THE DOUBLE TIME} AXIS

The paper [3] considered the following causal and unstable system $P_{h}: L_{\text {loc }}^{2}(\mathbb{R}) \rightarrow L_{\text {loc }}^{2}(R)$, defined by the convolution: ${ }^{1}$

$$
P_{h}: u \mapsto y: y(t)=\int_{-\infty}^{\infty} h(t-\tau) u(\tau) d \tau=(h * u)(t),
$$

where $h(t)=\exp (t)$ for $t \geq 0$ and 0 otherwise. This system has transfer function $\frac{1}{s-1}$. The interconnection of $P_{h}$ with a controller $C$ implementing negative unity feedback with gain greater than one as in Figure 1 was considered. It was shown [3] that if such a closed loop is considered to be well

\footnotetext{
W. Bian: School of Electronics and Computer Science University of Southampton, Southampton SO17 1BJ, United Kingdom wbdecs.soton.ac.uk

M. French: School of Electronics and Computer Science University of Southampton, Southampton SO17 1BJ, United Kingdom mcf@ecs.soton.ac.uk

H. Pillai: Department of Electrical Engineering, Indian Institute of Technology, Bombay, Indian hp@ee.iitb.ac. in

${ }^{1} f \in L_{\text {loc }}^{2}(\mathbb{R})$ if for all compact $\Omega \subset \mathbb{R},\left.f\right|_{\Omega} \in L^{2}(\Omega)$.
}

posed and stable (in the sense that the (single valued) map $\Pi: L^{2}\left(\mathbb{R}_{+}\right) \rightarrow L^{2}\left(\mathbb{R}_{+}\right)$defined by

$$
\Pi:\left(\begin{array}{c}
u_{0} \\
y_{0}
\end{array}\right) \mapsto\left(\begin{array}{l}
u_{1} \\
y_{1}
\end{array}\right)
$$

exists (and necessarily has finite induced norm)), then necessarily the classical $L^{2}(\mathbb{R})$ graph of $P_{h}$ is closed. It was further shown in [3] that the trajectories

$$
\begin{gathered}
\tilde{u}(t)=\left\{\begin{array}{ll}
\exp (-t) & t \geq 0 \\
0 & t<0
\end{array},\right. \\
\tilde{y}(t)= \begin{cases}-\frac{1}{2} \exp (-t) & t \geq 0 \\
-\frac{1}{2} \exp (t) & t<0\end{cases}
\end{gathered}
$$

can be obtained as the limit of a sequence of trajectories lying in the $L^{2}(\mathbb{R})$ graph of $P_{h}$, which is a contradiction since this solution does not satisfy the relation (2.1). This is a contradiction, and hence one is led to the conclusion that this system is not stabilizable under the conventional definitions. Furthermore it can be shown [3] that if $w_{0}=(\tilde{u}, \tilde{y})^{T}$ acts as the disturbance to the closed loop $\left[P_{h}, C\right]$, where $C$ is negative unity feedback in Figure 1, then there is no solution $w_{1}=\left(u_{1}, y_{1}\right)^{T}$.

The approach considered in [6] identifies the operator $P_{h}$ defined by $(2.1)$ with its $L^{2}(\mathbb{R})$ closure $\bar{P}_{h}$. In this case, the closure exists and is the (stable) anti-causal operator $\bar{P}_{h}=$ $P_{g}: L^{2}(\mathbb{R}) \rightarrow L^{2}(\mathbb{R})$, where

$$
P_{g}: u \mapsto y: y(t)=\int_{-\infty}^{\infty} g(t-\tau) u(\tau) d \tau=(g * u)(t),
$$

where $g(t)=-\exp (t)$ for $t \leq 0$ and zero otherwise. In [3], the identification of these two input-output systems is interpreted as 'more or less amount[ing] to abandoning any notion of causality', and it was stated that 'this is not a natural option, however, if the direction of time is well-defined'. It has the additional problem that $\bar{P}_{h}$ is stable, whose response on the bounded input $u(t)=1$ if $0 \leq t \leq 1, u(t)=0$ otherwise is the bounded output signal:

$$
y(t)= \begin{cases}0 & \text { if } t>1, \\ 1-\exp (1-t), & \text { if } 0 \leq t \leq 1 \\ \exp (t)-\exp (1+t), & \text { if } t<0\end{cases}
$$

We view this as problematic, since $P_{h}$ itself is defined as an operator $L^{2}(\mathbb{R}) \rightarrow L_{\text {loc }}^{2}(\mathbb{R})$, with the following unbounded output response to the above input:

$$
y(t)= \begin{cases}\exp (t)-\exp (1+t) & \text { if } t>1, \\ 1-\exp (t), & \text { if } 0 \leq t \leq 1, \\ 0, & \text { if } t<0 .\end{cases}
$$

These problems are only avoided in the case whereby the input-output operator has causal closure. In the discrete 
setting, the class of such transfer functions has been precisely characterised in [4] as the class of all Smirnoff functions; a class which includes all causal stable operators and excludes all causal unstable operators (as in the example considered); thus indicating that intrinsic difficulties with the input-output theory over $\mathbb{R}$.

The paradox can therefore be summarized as the inability to consider the system defined by the operator $P_{h}$ as both causal and stabilizable. Using the conventional definitions, one is limited to considering operators with causal closure.

We now consider a behavioural approach to this problem: seeking an alternative way to naturally define a system from the underlying operator $P_{h}$, which in turn does permit us to consider the system to be both causal and stabilizable.

\section{BehaVioural DEFINITIONS}

In this section we follow the development of [1] where this approach was used to produce a trajectory level behavioural approach to the gap metric and robust stability (see also [2]). Let $\mathcal{T}$ denote the time set, taken throughout to be either $\mathbb{Z}$ or $\mathbb{R}$, and let $\mathcal{T}_{+}=\mathbb{N}$ if $\mathcal{T}=\mathbb{Z}$ and $\mathcal{T}_{+}=\mathbb{R}_{+}$if $\mathcal{T}=\mathbb{R}$. For $n \geq 1$, an $n$-valued behaviour $\mathcal{B}$ is a subset of the set of all maps $\mathcal{T} \mapsto \mathbb{R}^{n}$, i.e. $\mathcal{B} \subset\left\{w: \mathcal{T} \rightarrow R^{n}\right\}$. The shift operator $\sigma_{t}, t \in \mathcal{T}$ is defined: $\sigma_{t} w(\cdot)=w(\cdot+t)$.

Definition 3.1: Let $\mathcal{B}$ be a behaviour. Then:

1) $\mathcal{B}$ is said to be linear if $\mathcal{B}$ is a vector space.

2) $\mathcal{B}$ is said to be shift invariant (time invariant) if $w \in \mathcal{B}$ implies $\sigma_{t} w \in B$ for all $t \in \mathcal{T}$.

Smooth differential behaviours are linear, shift invariant, continuous-time behaviours which can be expressed as the kernel of a differential operator, ie. those for which there exists a polynomial valued matrix $R$ s.t. that

$$
\mathcal{B}=\left\{w \in C^{\infty} \mid R\left(\frac{d}{d t}\right) w=0\right\} .
$$

Observe that in this note we will be interested in nondifferential/difference behaviours, for example, systems incorporating a time delay.

Definition 3.2: A behaviour $\mathcal{B}$ is said to have memory $l \geq$ 0 if for any $w_{1}, w_{2} \in \mathcal{B}$ with $\left.w_{1}\right|_{[a, a+l]}=\left.w_{2}\right|_{[a, a+l]}$ and $a \in \mathcal{T}$, the trajectory

$$
w_{3}(t)= \begin{cases}w_{1}(t) & \text { if } t \leq a \\ w_{2}(t) & \text { if } t \geq a\end{cases}
$$

also lies in $\mathcal{B}$.

If a behaviour has memory $0 \leq l<\infty$ it is said to have finite memory, if $l=0$ then it is memoryless. Note that a nonmemoryless continuous time differential behaviour has finite memory, and $l>0$ can be taken to be arbitrarily small; a discrete time behaviour also has finite memory, and here $l \geq 0$ depends on the system order. The minimal memory $l_{0} \geq 0$ of a behaviour $\mathcal{B}$ is the largest number s.t. $\mathcal{B}$ has memory $l$ for all $l>l_{0}$. Note that the minimum is not necessarily attained.

The standard definition of autonomy is that behaviour $\mathcal{B}$ is said to be autonomous if for any $w_{1}, w_{2} \in \mathcal{B},\left.w_{1}\right|_{(-\infty, 0]}=$ $\left.w_{2}\right|_{(-\infty, 0]}$ implies $w_{1}=w_{2}$. We relax this definition as follows:

Definition 3.3: A behaviour $\mathcal{B}$ with minimal memory $l_{0} \geq$ 0 is said to be autonomous if for any $w_{1}, w_{2} \in \mathcal{B}$, and any interval $V$ of length greater than $l_{0},\left.w_{1}\right|_{V}=\left.w_{2}\right|_{V}$ implies $\left.w_{1}\right|_{\mathcal{T}}=\left.w_{2}\right|_{\mathcal{T}}$.

Non-autonomy of a behaviour with finite memory is thus just the existence of a trajectory in the behaviour whose support has complement containing an interval of length greater than $l_{0}$, eg. a compactly supported trajectory.

\section{Stability}

Stability is determined by the signal spaces involved. We will consider the spaces $L^{p}\left(\mathcal{T}, \mathbb{R}^{n}\right)$ with $0 \leq p \leq \infty$. In the case when $\mathcal{T}=\mathbb{R}$, it is the standard $L^{p}$ spaces such as $L^{2}\left(\mathbb{R}, \mathbb{R}^{n}\right)$ and $L^{\infty}\left(\mathbb{R}, \mathbb{R}^{n}\right)$ for continuous-time signals. In the case when $\mathcal{T}=\mathbb{Z}$, it becomes the standard $l^{p}$ spaces used for discrete signals.

Given a general normed signal space (say) $Y$ of signals from $\mathcal{T}$ or $\mathcal{T}_{+}$to $\mathbb{R}^{n}$, the corresponding extended space $Y_{e}$ is defined as:

$$
Y_{e}=\left\{y: \mathcal{I} \rightarrow \mathbb{R}^{n}: T_{\tau} y \in Y \text { for all } \tau \in \mathcal{I}_{+}\right\},
$$

where $\mathcal{I}=\mathcal{T}$ or $\mathcal{T}_{+}$subject to on which set the space $Y$ is defined, and $T_{\tau}$ is the truncation operator, that is $\left(T_{\tau} y\right)(t)=$ $y(t)$ for $t \leq \tau$ and 0 for $t>\tau$.

Since this section, the behaviours considered will be restricted to be within the extended signal spaces $L_{e}^{p}:=$ $L_{e}^{p}\left(\mathcal{T}, \mathbb{R}^{n}\right), 1 \leq p \leq \infty$, i.e., $L^{p}$ behaviours or subsets of $L_{e}^{p}$. As a shorthand we denote by $X=L^{p}\left(\mathcal{T}_{+}\right)=$: $L^{p}\left(\mathcal{T}_{+}, \mathbb{R}^{n}\right), 1 \leq p \leq \infty$. So $X_{e}=L_{e}^{p}\left(\mathcal{T}_{+}\right)$. We remark that when the results do not need a normed structure on the signal spaces, our discussions and definitions also remain valid for $C^{\infty}$ behaviours (with $X=C^{\infty}\left(\mathcal{T}_{+}\right)$).

Recall that an operator $P: X_{e} \rightarrow Y_{e}$ is said stable is $P(X) \subset Y . P$ is called causal if $T_{\tau} P u=T_{\tau} P T_{\tau} u$ for all $\tau \geq 0$ and $u \in X_{e}$, given $X_{e}, Y_{e}$ two signal spaces.

We generalise the standard behavioural definition of stability for autonomous systems as follows:

Definition 4.1: An autonomous system $\mathcal{B}_{\text {aut }}$ is said to be $X$-stable if for any $w \in \mathcal{B}_{\text {aut }},\left.w\right|_{[0, \infty)} \in X$.

For non-autonomous systems, we adopt the following stability concept for behaviours with i/o partition (see [5]), which captures the notion of 'whatever the past, given a bounded future input, the future output is bounded':

Definition 4.2: A behaviour $\mathcal{B}$ with i/o partition $u \mid y$ is $X$ stable if for all $(u, y) \in \mathcal{B}$ with $\left.u\right|_{\mathcal{T}_{+}} \in X$ we have $\left.y\right|_{\mathcal{T}_{+}} \subset X$. When $X$ is given, throughout the paper we refer to the notion of ' $X$-stability' simply as 'stability'.

We now introduce a notion of uniform stability, which captures the property that in addition to stability, there is a uniform gain between future inputs and outputs when the past is zero. This notion of stability is closely related to the dissipativity descriptions of stability in [11], [7].

Definition 4.3: A linear behaviour with i/o partition $u \mid y$ is uniformly stable if

1) $\mathcal{B}$ is stable.

2) There exists a bounded operator $\Psi: X \rightarrow X$ such that for all $(u, y) \in \mathcal{B}$ such that $\left.u\right|_{\mathcal{T}_{+}} \in X,\left.(u, y)\right|_{\mathcal{T}_{-}}=0$ it follows that $y=\Psi(u)$.

Definition 4.4: A behaviour $\mathcal{B}$ is said to be stabilizable if for all $w_{1} \in \mathcal{B}$, there exists $w_{2} \in \mathcal{B}$ s.t. $\left.w_{1}\right|_{(-\infty, 0]}=$ $\left.w_{2}\right|_{(-\infty, 0]}$ and $\left.w_{2}\right|_{[0, \infty)} \in X$. 


\section{INTERCONNECTIONS}

We are primarily interested in the standard feedback interconnections shown in Figures 1 and 2.

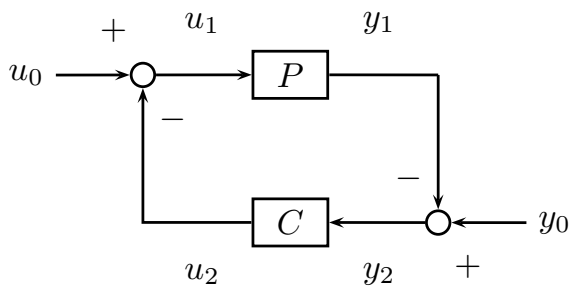

Fig. 1. The closed-loop.

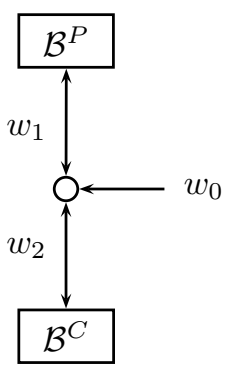

Fig. 2. The interconnected behaviours: $w_{i}=\left(u_{i}, y_{i}\right)^{T}, i=0,1,2$.

Definition 5.1: Given a plant behaviour $\mathcal{B}^{P}$, a controller behaviour $\mathcal{B}^{C}$ and interconnection behaviour $\mathcal{B}^{I}$ :

$$
\mathcal{B}^{I}=\left\{\left(w_{0}, w_{1}, w_{2}\right)^{T} \in X_{e} \mid w_{0}=w_{1}+w_{2}\right\}
$$

we define the closed loop behaviour $\mathcal{B}^{P{ } C} C$ as follows:

$$
\mathcal{B}^{P \wedge_{I} C}=\left\{\left(w_{0}, w_{1}, w_{2}\right)^{T} \in \mathcal{B}^{I} \mid w_{1} \in \mathcal{B}^{P}, w_{2} \in \mathcal{B}^{C}\right\} .
$$

To ensure uniqueness of solutions of the closed loop (modulo the autonomous part of the behaviour) we adopt the following definition: be

Definition 5.2: The extended graph $\mathcal{G}_{\mathcal{B}}$ of $\mathcal{B}$ is defined to

$\mathcal{Z}_{\mathcal{B}}:=\left\{\left.w \in \mathcal{B}\right|_{\mathbb{R}_{+}}|v \in \mathcal{B}, w|_{\mathbb{R}_{+}}=\left.v\right|_{\mathbb{R}_{+}},\left.v\right|_{(-\infty, 0]}=0\right\}$.

Definition 5.3: Given a plant behaviour $\mathcal{B}^{P}$, a controller behaviour $\mathcal{B}^{C}$ and interconnection behaviour $\mathcal{B}^{I}$ (5.4), the behaviour $\mathcal{B}^{P \wedge_{I} C}$ is said to be well posed if

$$
X_{e}=\mathcal{Z}_{\mathcal{B}^{P}} \oplus \mathcal{Z}_{\mathcal{B}^{C}}
$$

This captures the idea that for the interconnection of behaviours with zero past, ' $w_{0}$ is an input, and for any input $w_{0}$, there exist unique internal signals $w_{1}, w_{2}$ '.

Definition 5.4: A controller behaviour $\mathcal{B}^{C}$ is said to be a stabilizing controller for a plant behaviour $\mathcal{B}^{P}$ if $\mathcal{B}^{P \wedge{ }_{I} C}$ is stable.

\section{The DOUble TIME AXIS PARADOX IN THE BEHAVIOURAL CONTEXT}

Let us consider the simplest behaviour associated with the operator (2.1):

$$
\mathcal{B}_{\mathrm{io}}=\left\{\left(\begin{array}{l}
u \\
y
\end{array}\right) \in L_{\mathrm{loc}}^{2}(\mathbb{R}) \times L_{\mathrm{loc}}^{2}(\mathbb{R}): y=P_{h} u\right\} .
$$

By linearity the input-output model (2.1) enforces $u=0$ implies $y=0$, hence $\mathcal{B}_{\mathrm{io}}$ has no non-trivial autonomous sub-behaviour. By the natural inclusion of such autonomous sub-behaviours, and with the corresponding relaxations of the notions of causality, well-posedness and stability, the example can be reconsidered as follows.

Let $\mathcal{B}^{P_{h}}$ denote the smallest differential behaviour containing $\mathcal{B}_{\text {io }}$. It can be observed that $\mathcal{B}^{P_{h}}$ can be expressed by the following (minimal) kernel representation:

$$
\mathcal{B}^{P_{h}}=\left\{w \in L_{\text {loc }}^{2}(\mathbb{R}) \mid[-1 s-1] w=0\right\},
$$

where $s=\frac{d}{d t}$, where solutions are interpreted in the weak sense in $L_{\text {loc }}^{2}(\mathbb{R})$ (rather than $L_{e}^{2}(\mathbb{R})$ ) to avoid any possible implicit imposition of a time-direction. For any $y_{0} \in \mathbb{R}, u \in$ $L_{\text {loc }}^{2}(\mathbb{R})$, write

$$
L\left(y_{0}, u\right)=y_{0} \exp (t)+\int_{-\infty}^{t} \exp (t-\tau) u(\tau) d \tau .
$$

The behaviour $\mathcal{B}^{P_{h}}$ can be explicitly expressed as:

$$
\mathcal{B}^{P_{h}}=\left\{\left(\begin{array}{l|l}
u \\
y
\end{array}\right) \in L_{\mathrm{loc}}^{2}(\mathbb{R}) \mid \begin{array}{c}
u \in L_{\text {loc }}^{2}(\mathbb{R}), y_{0} \in \mathbb{R}, \\
y=L\left(y_{0}, u\right)
\end{array}\right\} .
$$

In terms of the definitions given in this paper, this behaviour is indeed stabilizable, and negative unity feedback with a gain greater than one provides a well-posed stabilizing interconnection: if

$$
\mathcal{B}^{C}=\left\{w \in L_{e}^{2}\left(\mathbb{R}_{+}\right) \mid w=(u, y)^{T}, u(t)=-k y(t)\right\}
$$

then $\mathcal{B}^{P_{h} \wedge_{I} C}$ is (uniformly) stable for $k>1$. It is important to observe that

$$
\begin{aligned}
\left(\begin{array}{c}
\tilde{u} \\
\tilde{y}
\end{array}\right) & =\left(\begin{array}{c}
\tilde{u} \\
\exp (t)+\int_{-\infty}^{t} \exp (t-\tau) \tilde{u}(\tau) d \tau
\end{array}\right) \\
& \in \mathcal{B}^{P_{h}} \cap L^{2}(\mathbb{R}),
\end{aligned}
$$

since $(\tilde{u}, \tilde{y})^{T}$ can be explained by the sum of the unforced solution

$$
(0, \exp (\cdot))^{T}
$$

and the forced solution

$$
\left(\tilde{u}, \int_{-\infty}^{t} \exp (t-\tau) \tilde{u}(\tau) d \tau\right)^{T} .
$$

It is important to observe that the approach developed in this paper provides an alternative approach to addressing the classical problems of doubly infinite time axis. The approach taken here is perhaps half way between a double and a half line time axis, in that signals are defined over the whole of $\mathbb{R}$, but stability notions are related boundedness of signals when restricted to $\mathbb{R}_{+}$. Only in the case of uniform stability do we consider an induced norm and a zero past. The notion of wellposedness again restricts attention to the sub-behaviour with a zero past, and importantly does not impose uniqueness of solutions: $\left(w_{0}, w_{1}, w_{2}\right),\left(w_{0}, v_{1}, v_{2}\right) \in \mathcal{B}^{P_{h} \wedge C}$ does not imply $\left(w_{1}, w_{2}\right)=\left(v_{1}, v_{2}\right)$ in general.

In common with these other approaches to resolving the socalled 'Georgiou-Smith' paradox, the procedure of identifying the convolution system (2.1) with the smallest differential 
behaviour containing the same input-output pairs also identifies the same behaviour $\mathcal{B}^{P}=\mathcal{B}^{P_{h}}=\mathcal{B}^{P_{g}}$ to the anticausal input-output system (2.2) as it is easily verified that $\mathcal{B}^{P}$ contains all trajectories $(u, y) \in L_{\text {loc }}^{2}$ satisfying $(2.2)^{2}$. However, the consideration of $\mathcal{B}^{P}$ permits us to maintain a sensible notion of causality as follows:

Definition 6.1: A behaviour $\mathcal{B}$ with input-output partition $(u, y)$ is said to be causal if

$$
T_{\tau} u_{1}=T_{\tau} u_{2} \quad \Longrightarrow \quad T_{\tau} \mathcal{B}_{u_{1}}=T_{\tau} \mathcal{B}_{u_{2}}
$$

where $\mathcal{B}_{u}=\{w \in \mathcal{B} \mid \exists y$ s.t. $w=(u, y) \in \mathcal{B}\}$.

This can be interpreted as stating that the set of all past trajectories which can be generated from a particular past input cannot be affected by changing the future input, and represents a generalisation of the notion of a causal operator (where the non-uniqueness of the output given the input is suitably accounted for). We can now observe that $\mathcal{B}^{P}$ indeed preserves causality, and we have thus arrived at a position whereby we can consider a suitable treatment of the system (2.1) in which the physical object under study can be thought of as causal and stabilizable. We find the explanation of trajectories of the system as a combination of an autonomous unforced sub-behaviour and a causal input to be more in line with physical thinking than the interpretation of the trajectories arising from a non-causal input to a single valued operator.

\section{REFERENCES}

[1] W. Bian, M. French and H. Pillai, An intrinsic behavioural approach to the gap metric, Proc. 44th IEEE CDC and ECC'05, Spain, 2005, 1553-1558.

[2] W. Bian, M. French and H. Pillai, An intrinsic behavioural approach to the gap metric, SIAM Journal of Control and Optimization, 47(4):19391960, 2008.

[3] T. Georgiou and M. C. Smith,Intrinsic difficulties in using the doublyinfinte time axis for input-output control theory, IEEE Trans. Auto. Control, 40(1995), 516-518

[4] B. Jacob and J. Partington, Graphs, closability, and causality of linear time-invariant discrete-time systems. Int. J. Control, 73(2000) (11) 1051-1060.

[5] J. W. Polderman and J. C. Willems, Introduction to Mathematical Systems Theory, Springer, 1997

[6] P.M. Mäkilä, On three puzzles in robust control IEEE Trans. Auto. Control, 45(2000), 552-556.

[7] H. L. Trentelman and J. C. Willems, Synthesis of dissipative systems using quadratic differential forms: Part II, IEEE Trans. Auto. Control, 47(2002), $70-86$

[8] J. C. Willems, From time series to linear system - Part I. Finite dimensional linear time invariant systems, Automatica, 22(1986), 561580

[9] J. C. Willems, From time series to linear system - Part II. Exact modelling, Automatica, 22(1986), 675-694

[10] J. C. Willems, From time series to linear system - Part III. Approximate modelling, Automatica, 23(1987), 87-115

[11] J. C. Willems and H. L. Trentelman, Synthesis of dissipative systems using quadratic differential forms: Part I, IEEE Trans. Auto. Control, 47(2002), 53-69

\footnotetext{
${ }^{2}$ Importantly however, $\mathcal{B}^{P}$ also includes unbounded trajectories such as $(0, \exp (\cdot))^{T}$ which are neither of the form $\left(u, P_{h} u\right)^{T}$ nor $\left(u, P_{g} u\right)$.
} 